\title{
Elemental abundance analyses with DAO spectrograms
}

\section{The superficially normal B and A stars $\alpha$ Draconis, $\tau$ Herculis, $\gamma$ Lyrae, and HR 7926*}

\author{
Saul J. Adelman ${ }^{1,2}$, H. Caliskan ${ }^{3}$, D. Kocer ${ }^{3}$, H. Kablan ${ }^{3}$, K. Yüce ${ }^{4}$, and S. Engin ${ }^{4}$ \\ 1 Department of Physics, The Citadel, 171 Moultrie Street, Charleston, SC 29409, USA \\ 2 Guest Investigator, Dominion Astrophysical Observatory, Herzberg Institute of Astrophysics, \\ National Research Council of Canada, 5071 W. Saanich Road, Victoria V8X 4M6 Canada \\ 3 Department of Astronomy and Space Sciences, Istanbul University, 34452 University, Istanbul, Turkey \\ ${ }^{4}$ Department of Astronomy and Space Sciences, Science Faculty, Ankara University, 06100, Tandoğan, Ankara, \\ Turkey
}

Received 26 January 2001 / Accepted 15 March 2001

\begin{abstract}
Elemental abundances analyses of the superfically normal B and A stars $\alpha$ Dra (A0 III), $\tau$ Her (B5 IV), $\gamma$ Lyr (B9 III), and HR 7926 (B8 II-III) are performed consistent with previous studies of this series using spectrograms obtained with Reticon and CCD detectors. Comparisons of the first two analyses with those of the same stars performed earlier in this series which used mostly coadded photographic plates show the general consistency of the derived elemental abundances. A slight increase in the adopted effective temperature produces a corresponding increase in the derived abundances. In these stars the $\mathrm{He} / \mathrm{H}$ ratios are found to be close to solar. Except for $\gamma$ Lyr the metals show for the most part marginally subsolar abundance values. But this star has Al, $\mathrm{Ca}, \mathrm{Sc}$, and $\mathrm{Sr}$ abundances that are substantially underabundant as well as other underabundant values.
\end{abstract}

Key words. stars: abundances - stars: individual: $\alpha$ Dra - stars: individual: $\tau$ Her - stars: individual: $\gamma$ Lyr stars: individual: HR 7926

\section{Introduction}

Paper XXIV (Adelman et al. 2001) summarized the improvements in the analyses techniques in this series which began in 1987 and showed by comparing the results of two new analyses based on Reticon and CCD spectrograms with those made previously using based on coadded photographic spectrograms that the discrepancies were for the most part small and hence the analyses of this series are quite consistent. In this paper we continue this process with new studies of $\alpha$ Dra and $\tau$ Her as well as study the late B stars $\gamma$ Lyr and HR 7926 using CCD spectrograms.

$\alpha$ Dra (Thuban, HD 123299, HR 5291), spectral type A0 III, and $\tau$ Her (HD 147394, HR 6092), spectral type B5 IV, were analyzed by Adelman (1994, Paper XIII) and Adelman (1988, Paper III), respectively. The former is a

\footnotetext{
Send offprint requests to: Saul J. Adelman, e-mail: adelmans@citadel.edu

* Table 5 is only available in electronic form at the CDS via anonymous ftp to cdsarc.u-strasbg.fr (130.79.128.5) or via

http://cdsweb.u-strasbg.fr/cgi-bin/qcat?J/A+A/371/1078
}

slightly metal poor star which is also a single-lined spectroscopic binary. The last study was based on coadded photographic region spectrograms supplemented by eight Reticon spectra, most of which are reanalyzed in this paper. The derived abundances of the later star except for perhaps $\mathrm{Ni}$ are solar. Its analysis was based entirely on coadded photographic spectra.

$\gamma$ Lyr (HD 176437, HR 7178, 14 Lyr) was discovered by Mitchell (1909) to be a spectroscopic binary. Abt \& Morell (1995) classify it as a B9 III star with $v \sin i=60 \mathrm{~km} \mathrm{~s}^{-1}$. HR 7926 (HD 197392) was classified by Cowley (1972) as B8 II-III. Wolff (1978) found it was a spectroscopic binary with a period greater than 80 days and $v \sin i=25 \mathrm{~km} \mathrm{~s}^{-1}$. Our studies of $\gamma$ Lyr and HR 7926 increase the number of stars showing some rotation and extend the range of luminosity for this series.

\section{The spectra}

For each star we obtained 17 Dominion Astrophysical Observatory (DAO) $2.4 \AA \mathrm{mm}^{-1}$ Reticon or site $2 \mathrm{CCD}$ spectrograms with a typical signal-to-noise ratio of 200 
and a wavelength coverage of 67 or $63 \AA$, respectively. The central wavelengths between $\lambda 3830$ and $\lambda 4740$ had $55 \AA$ offsets. For all four stars $20 \AA \mathrm{mm}^{-1}$ DAO spectrograms containing the $\mathrm{H} \gamma$ region and $2.4 \AA \mathrm{mm}^{-1}$ spectra centered at $\lambda 4905, \lambda 5015$, and $\lambda 5070$ were obtained as well as at $\lambda 5840$ for HR 7926. Further $2.4 \AA \mathrm{mm}^{-1}$ spectra using the new thinned site 4 CCD with a wavelength coverage of $147 \AA$, centered at $\lambda 4726$ were obtained for $\alpha$ Dra and $\tau$ Her and at $\lambda 4864$ for $\alpha$ Dra. The exposures were flat fielded with the exposures of an incandescent lamp located in the Coudé mirror train as viewed through a filter to eliminate first order light except for the exposures in first order at $\lambda 5840$ where no filter was used. A central stop removed light from the beam in the same manner as the secondary mirror of the telescope. The spectra were rectified using the interactive computer graphics program REDUCE (Hill et al. 1982). A correction of 3.5\% was applied for scattered light in the dispersion direction (Gulliver et al. 1996).

Rotational profiles were fit through the stellar metal lines of our four stars except for strong He I lines for which Lorentzian profiles were used. Rotational velocity estimates based on clearly single medium strength lines near $\lambda 4481$ are $25 \mathrm{~km} \mathrm{~s}^{-1}$ for $\alpha$ Dra, $37 \mathrm{~km} \mathrm{~s}^{-1}$ for $\tau$ Her, $70 \mathrm{~km} \mathrm{~s}^{-1}$ for $\gamma \mathrm{Lyr}$, and $26 \mathrm{~km} \mathrm{~s}^{-1}$ for HR 7926. For some observations, the line profiles of $\tau$ Her are asymmetric, which is consistent with it exhibiting non-radial pulsations (see, e.g., Campos \& Smith 1980). Paper XIII found the same value of $v \sin i$ for $\alpha$ Dra while for $\tau$ Her Paper III gives $32 \mathrm{~km} \mathrm{~s}^{-1}$ which is less than that for this paper. Our value may reflect better determined line profiles.

The stellar lines were identified with the general references A Multiplet Table of Astrophysical Interest (Moore 1945) and Wavelengths and Transition Probabilities for Atoms and Atomic Ions, Part 1 (Reader \& Corliss 1980) as well as Huldt et al. (1982) for Ti II, Iglesias \& Velasco (1964) for Mn II, and Dworetsky (1971); Johansson (1978); Guthrie (1985); and Adelman (1987) for Fe II. For $\alpha$ Dra, lines of H I, He I, C I, C II, O I, Mg I, Mg II, Al I, Al II, Si I, Si II, S II, Ca I, Ca II, Sc II, Ti II, V II, Cr I, Cr II, Mn I, Mn II, Fe I, Fe II, Ni II, Sr II, and Ba II were identified in Paper XIII. These species except for Mn I, whose identification was based on only one weak line, were also seen in this study. For $\tau$ Her the analysis in Paper III and this study found lines of H I, He I, C II, N II, O II, Mg II, Al III, Si II, Si III, S II, Ca II, Ti II, Cr II, Fe II, Fe III, and Ni II. The only newly found species was Al II represented by one line. In the spectrum of $\gamma$ Lyr, we found lines of H I, He I, C II, O I, Mg I, Mg II, Al I, Al II, Si II, Ca II, Sc II, Ti II, V II, Cr II, Mn II, Fe I, Fe II, Ni II, and Sr II while HR 7926 shows lines of H I, He I, C II, N II, O I, Mg II, Al II, Al III, Si II, Si III, S II, Ca II, Ti II, Cr II, Mn II, Fe II, Fe III, and Ni II.

The radial velocities come from comparisons of the stellar and laboratory wavelengths after corrections were applied for the Earth's orbital velocity. For $\alpha$ Dra, $\gamma$ Lyr, and HR 7926, which are single-lined spectroscopic
Table 1. Radial velocity measurements

\begin{tabular}{|c|c|c|}
\hline $\begin{array}{c}\text { central } \\
\lambda(\AA)\end{array}$ & $\begin{array}{c}\text { Heliocentric } \\
\text { Julian date }\end{array}$ & $\begin{array}{r}R V \\
\left(\mathrm{~km} \mathrm{~s}^{-1}\right)\end{array}$ \\
\hline \multicolumn{3}{|l|}{$\alpha$ Dra } \\
\hline 4190 & 2447333.740 & -4.4 \\
\hline 4245 & 2447335.718 & -20.1 \\
\hline 3860 & 2448475.700 & -43.3 \\
\hline 4630 & 2448479.685 & -44.8 \\
\hline 4685 & 2448480.682 & -44.6 \\
\hline 4740 & 2448796.821 & -1.5 \\
\hline 4355 & 2449141.737 & -40.7 \\
\hline 4465 & 2450168.062 & -35.9 \\
\hline 4300 & 2450168.839 & -36.4 \\
\hline 4410 & 2450306.014 & 32.5 \\
\hline 4080 & 2450307.011 & 40.1 \\
\hline 3915 & 2450309.011 & 52.3 \\
\hline 4520 & 2450529.061 & -36.5 \\
\hline 4905 & 2450589.836 & -39.9 \\
\hline 5070 & 2450592.987 & -40.0 \\
\hline 5015 & 2450593.984 & -38.8 \\
\hline 3970 & 2450653.998 & -25.1 \\
\hline 4025 & 2450654.992 & -21.3 \\
\hline 4135 & 2450655.817 & -17.8 \\
\hline 4575 & 2450658.996 & -8.3 \\
\hline 4726 & 2451609.001 & -50.7 \\
\hline 4864 & 2451660.724 & -29.8 \\
\hline \multicolumn{3}{|l|}{$\gamma \mathrm{Lyr}$} \\
\hline 4685 & 2450400.572 & -23 \\
\hline 4520 & 2450529.061 & -23 \\
\hline 4905 & 2450590.987 & -26 \\
\hline 5015 & 2450593.984 & -21 \\
\hline 4355 & 2450595.909 & -23 \\
\hline 4245 & 2450596.827 & -21 \\
\hline 4190 & 2450601.927 & -21 \\
\hline 5070 & 2450650.963 & -26 \\
\hline 4740 & 2450651.804 & -19 \\
\hline 3970 & 2450653.998 & -27 \\
\hline 4025 & 2450654.991 & -24 \\
\hline 4135 & 2450655.816 & -29 \\
\hline 4300 & 2450657.001 & -22 \\
\hline 4410 & 2450657.813 & -28 \\
\hline 4575 & 2450658.996 & -22 \\
\hline 4630 & 2450661.009 & -23 \\
\hline 4080 & 2450753.631 & -24 \\
\hline 4465 & 2450920.991 & -23 \\
\hline 3915 & 2451028.843 & -22 \\
\hline 3860 & 2451408.841 & -27 \\
\hline \multicolumn{3}{|l|}{ HR 7926} \\
\hline 4245 & 2449618.766 & -46 \\
\hline 4520 & 2449923.911 & -61 \\
\hline 4465 & 2450305.014 & 1 \\
\hline 4410 & 2450306.014 & -7 \\
\hline 4080 & 2450307.011 & -12 \\
\hline 4905 & 2450648.988 & -32 \\
\hline 5015 & 2450649.989 & -32 \\
\hline 4190 & 2450656.002 & -25 \\
\hline 4355 & 2450658.003 & -30 \\
\hline 4630 & 2450661.009 & -20 \\
\hline 4685 & 2450662.003 & -19 \\
\hline 4740 & 2450694.951 & 5 \\
\hline 4135 & 2450697.953 & -6 \\
\hline 3860 & 2450994.983 & 9 \\
\hline 4025 & 2451027.881 & -25 \\
\hline 4300 & 2451306.835 & 20 \\
\hline 4575 & 2451399.843 & 10 \\
\hline 5840 & 2451410.732 & -40 \\
\hline 5070 & 2451413.032 & -46 \\
\hline 3970 & 2451440.037 & -42 \\
\hline 3915 & 2451441.913 & -22 \\
\hline
\end{tabular}


Table 2. Effective temperature and surface gravity determinations

\begin{tabular}{|c|c|c|c|}
\hline Star & $\begin{array}{c}\text { deduced } \\
T_{\text {eff }}(\mathrm{K})\end{array}$ & $\begin{array}{r}\text { deduced } \\
\log g\end{array}$ & Method \\
\hline \multirow[t]{2}{*}{$\alpha$ Dra } & 9975 & 3.63 & Napiwotzki et al. (1993) with uvby $\beta$ photometry \\
\hline & 10000 & 3.60 & Spectrophotometry and $\mathrm{H} \gamma$ profile fitting, solar model, $\xi=0.0 \mathrm{~km} \mathrm{~s}^{-1}$ \\
\hline \multirow[t]{2}{*}{$\tau$ Her } & 15022 & 3.93 & Napiwotzki et al. (1993) with uvby $\beta$ photometry \\
\hline & 15000 & 4.10 & Spectrophotometry and $\mathrm{H} \gamma$ profile fitting, solar model, $\xi=0.0 \mathrm{~km} \mathrm{~s}^{-1}$ \\
\hline \multirow[t]{2}{*}{$\gamma$ Lyr } & 9674 & 2.67 & Napiwotzki et al. (1993) with uvby $\beta$ photometry \\
\hline & 9550 & 2.75 & Spectrophotometry and $\mathrm{H} \gamma$ profile fitting, solar model, $\xi=2.0 \mathrm{~km} \mathrm{~s}^{-1}$ \\
\hline \multirow[t]{2}{*}{ HR 7926} & 13306 & 3.42 & Napiwotzki et al. (1993) with uvby $\beta$ photometry \\
\hline & 13306 & 3.55 & Adjustment to $\log g$ to best fit the $\mathrm{H} \gamma$ profile \\
\hline
\end{tabular}

binaries, we list the radial velocities as they can be used to improve the orbit of the first star and to find the orbits of the other two stars. The mean radial velocity of $\tau$ Her is $=-16.3 \pm 4.1 \mathrm{~km} \mathrm{~s}^{-1}$. The individual values are not given for this non-radial pulsator. For $\alpha$ Dra we could not find any lines of the secondary. This also appears to be true for both $\gamma$ Lyr and HR 7926, but their Ca II H and $\mathrm{K}$ lines also have a sharper component, which could be interstellar.

\section{The abundance analyses}

Table 2 lists our effective temperature and surface gravity estimates with the last values for each star being those adopted. We began with the computer program of Napiwotzki et al. (1993) and the homogeneous uvby $\beta$ data of Hauck \& Mermilliod (1980). The uncertainties are about $\pm 150 \mathrm{~K}$ and \pm 0.2 dex (Lemke 1989). To refine these values we calculated synthetic spectra of the $\mathrm{H} \gamma$ regions from ATLAS9 LTE plane parallel model atmospheres (Kurucz 1993) with Program SYNTHE (Kurucz \& Avrett 1981) as well as the predicted fluxes with ATLAS9 for comparison with the observations which are from Adelman (1978) for $\alpha$ Dra and $\tau$ Her, from Schild et al. (1971) as modified by Breger (1976) for $\gamma$ Lyr, and from Kharitonov et al. (1997) for HR 7926. For $\alpha$ Dra the temperature is $25 \mathrm{~K}$ less and the surface gravity 0.15 dex less than the values of Paper XIII while for $\tau$ Her the temperature is $250 \mathrm{~K}$ greater and the surface gravity 0.45 dex greater than those used in Paper III which were based on ATLAS6 (Kurucz 1979) rather than ATLAS9 models (Kurucz 1993). Comparison of the predicted fluxes for a model atmosphere of HR 7926 with the photometric values of effective temperature and surface gravity with the observations of Kharitonov et al. (1997) show that the size of the Balmer jump is approximately correct, but that the observed Balmer continuum only crudely looks like the predictions is a substantial reason not to use these values. The photometry indicates that HR 7296 is slightly red- dened by $E(b-y)=0.027$ which must be allowed for in determining its values. A small adjustment in $\log g$ makes the spectral synthesis predictions agree with the observed $\mathrm{H} \gamma$ profile. HR 7926, which is classified as a higher luminosity star than $\gamma$ Lyr, has the greater surface gravity of the two stars. This is in accord with the determination of absolute magnitudes using parallaxes from Hipparcos (ESA 1997).

Paper XXIV indicates the changes in abundances due to a $100 \mathrm{~K}$ change in effective temperature and a 0.2 dex change in $\log g$ for $\phi$ Her. They are useful in estimating similar changes for stars of this paper.

The helium and metal abundances were determined using programs SYNSPEC (Hubeny et al. 1994) and WIDTH9 (Kurucz 1993), respectively, with metal line damping constants from Kurucz \& Bell (1995) or semiclassical approximations in their absence. Abundances from Fe I and II lines were derived for a range of possible microturbulences whose adopted values (Table 3 ) result in the derived abundances being independent of the equivalent widths $\left(\xi_{1}\right)$ and having a minimal scatter about the mean $\left(\xi_{2}\right)$ (Blackwell et al. 1982). For $\alpha$ Dra, we find $0.1 \mathrm{~km} \mathrm{~s}^{-1}$, which is the same as in Paper XIII. But for $\tau$ Her and HR 7926, we lack sufficient lines of any species which are beyond the linear part of the curve-of-growth to determine the microturbulence. Thus we use $0.0 \mathrm{~km} \mathrm{~s}^{-1}$, the canonical value, which appears to be appropriate for stars of similar effective temperature and surface gravity. This was done in Paper III for $\tau$ Her. For $\gamma$ Lyr, we find $0.8 \mathrm{~km} \mathrm{~s}^{-1}$ using all our Fe II lines. There are insufficient Fe I lines for this determination.

The helium abundances (Table 4 ) were found by comparing the line profiles with theoretical predictions which were convolved with the rotational velocity and the instrumental profile. For these stars the $\mathrm{He} / \mathrm{H}$ values are fairly consistent from line to line. For $\alpha$ Dra, He I $\lambda 4472$ is most discrepant and its profile suggests that it might be blended in its shortward wing. In addition lines at $\lambda 3868, \lambda 4143$, and $\lambda 5047$ were not used as they are too weak or blended. 
Table 3. Microturbulence determinations from Fe I and Fe II lines

\begin{tabular}{llcccccc}
\hline Star & Species & $\begin{array}{c}\text { Number } \\
\text { of lines }\end{array}$ & $\begin{array}{c}\xi_{1} \\
\left(\mathrm{~km} \mathrm{~s}^{-1}\right)\end{array}$ & $\log N / N_{\mathrm{T}}$ & $\begin{array}{c}\xi_{2} \\
\left(\mathrm{~km} \mathrm{~s}^{-1}\right)\end{array}$ & $\log N / N_{\mathrm{T}}$ & $g f$ values \\
\hline$\alpha$ Dra & Fe I & 54 & 0.4 & $-4.81 \pm 0.24$ & 0.3 & $-4.81 \pm 0.24$ & $\mathrm{MF}$ \\
& & 62 & 0.0 & $-4.77 \pm 0.27$ & 0.0 & $-4.77 \pm 0.27$ & $\mathrm{MF}+\mathrm{KX}$ \\
& \multirow{2}{*}{ Fe II } & 43 & 0.0 & $-4.74 \pm 0.17$ & 0.0 & $-4.74 \pm 0.17$ & $\mathrm{MF}$ \\
& & 71 & 0.0 & $-4.70 \pm 0.19$ & 0.0 & $-4.70 \pm 0.19$ & $\mathrm{MF}+\mathrm{KX}$ \\
& & & & & & \\
\multirow{2}{*}{$\gamma$ Lyr } & Fe II & 45 & 0.1 & & & & \\
& & adopted & 0.9 & $-4.90 \pm 0.19$ & 0.8 & $-4.89 \pm 0.19$ & $\mathrm{MF}+\mathrm{KX}$ \\
& & & & & & \\
\hline
\end{tabular}

$g f$ value references: $\mathrm{MF}=$ Fuhr et al. (1988), KX $=$ Kurucz \& Bell (1995).

Note: For $\xi_{1}$ and $\xi_{2}$ the abundances are found so that there is no trend of values for lines

of different equivalent widths and have minimum scatter about the mean, respectively.

Table 4. $\mathrm{He} / \mathrm{H}$ determinations

\begin{tabular}{|c|c|c|}
\hline Star & $\lambda(\AA)$ & $\mathrm{He} / \mathrm{H}$ \\
\hline \multirow[t]{6}{*}{$\alpha$ Dra } & 4026 & 0.08 \\
\hline & 4121 & 0.10 \\
\hline & 4388 & 0.10 \\
\hline & 4472 & 0.06 \\
\hline & 4713 & 0.09 \\
\hline & 4921 & 0.09 \\
\hline average & & $0.09 \pm 0.02$ \\
\hline \multirow[t]{13}{*}{$\tau$ Her } & 3868 & 0.10 \\
\hline & 4009 & 0.12 \\
\hline & 4026 & 0.10 \\
\hline & 4121 & 0.12 \\
\hline & 4169 & 0.10 \\
\hline & 4388 & 0.10 \\
\hline & 4437 & 0.10 \\
\hline & 4472 & 0.10 \\
\hline & 4713 & 0.11 \\
\hline & 4922 & 0.11 \\
\hline & 5015 & 0.12 \\
\hline & 5047 & 0.13 \\
\hline & average & $0.11 \pm 0.01$ \\
\hline \multirow[t]{7}{*}{$\gamma$ Lyr } & 4026 & 0.09 \\
\hline & 4143 & 0.12 \\
\hline & 4388 & 0.11 \\
\hline & 4472 & 0.11 \\
\hline & 4713 & 0.10 \\
\hline & 4922 & 0.10 \\
\hline & average & $0.10 \pm 0.01$ \\
\hline \multirow[t]{12}{*}{ HR 7926} & 4009 & 0.10 \\
\hline & 4026 & 0.10 \\
\hline & 4121 & 0.10 \\
\hline & 4143 & 0.10 \\
\hline & 4388 & 0.10 \\
\hline & 4437 & 0.10 \\
\hline & 4472 & 0.10 \\
\hline & 4713 & 0.10 \\
\hline & 4921 & 0.11 \\
\hline & 5015 & 0.10 \\
\hline & 5047 & 0.12 \\
\hline & average & $0.10 \pm 0.01$ \\
\hline
\end{tabular}

In the spectrum of $\tau$ Her, the lines at $\lambda 3872, \lambda 3926, \lambda 3964$, and $\lambda 4143$ were not used. Further $\lambda 4121$ and $\lambda 5047$ have profiles which suggest they might be blended. In the spectrum of $\gamma$ Lyr, the lines at $\lambda 3868, \lambda 3926$, and $\lambda 4437$ were found, but not analyzed due to being blended or weakness. For HR 7926 lines seen but not used include $\lambda 3868$, $\lambda 3872, \lambda 3926, \lambda 3964, \lambda 4169$, and $\lambda 5840$ with the LTE result for the last line much larger than those of other lines. To convert $\log N / N_{\mathrm{T}}$ values to $\log N / H$ values $-0.04 \mathrm{dex}$, -0.05 dex, -0.05 dex, and -0.04 dex were added to values for $\alpha$ Dra, $\tau$ Her, $\gamma$ Lyr and HR 7926, respectively.

Table 5, the analyses of the metal line spectra, contains for each line the multiplet number (Moore 1945), the laboratory wavelength, the logarithm of the $g f$-value and its source, the equivalent width in $\mathrm{m} \AA$ as observed, and the deduced abundance. Source references are given at the end of this table. Letters are used in place of multiplet numbers to indicate sources other than Moore (1945): $\mathrm{D}=$ Dworetsky (1971), I = Iglesias \& Velasco (1964), and $\mathrm{J}=$ Johansson (1978).

\section{Discussion}

Table 6 compares the results of these studies of $\alpha$ Dra and of $\tau$ Her with those from Papers XIII and III, respectively. For $\alpha$ Dra most of the changes were small. For four elements with lines from two species, the agreement between the species improved while for two other elements, it became slight worse. The largest abundance change was for Ba increasing by 0.35 dex and the next largest was for $\mathrm{S}$ by 0.25 dex which are based on 2 and 1 lines, respectively. For $\tau$ Her there is a general tendency to increase the abundances by about 0.1 dex due to the increase in temperature. For $\mathrm{Ti}$ and $\mathrm{Ni}$ there is a much greater change, but this is due to the measurement of weak lines. These analyses and those of $v$ Her and $\phi$ Her by Adelman et al. (2001) by demonstrating that the abundances based on at least a few metal lines were little changed in the newer 
Table 6. Comparison of results $(\log N / \mathrm{H})$

\begin{tabular}{lcc}
\hline$\alpha$ Dra & & \\
Species & Paper XIII & This paper \\
\hline He I & -1.04 & -1.06 \\
C I & -3.91 & -3.78 \\
C II & -3.51 & -3.67 \\
O I & -3.49 & -3.50 \\
Mg I & -4.61 & -4.54 \\
Mg II & -4.65 & -4.70 \\
Al I & -6.07 & -6.24 \\
Al II & -5.76 & -5.83 \\
Si II & -4.89 & -4.96 \\
S II & -4.60 & -4.35 \\
Ca I & -6.28 & -6.25 \\
Ca II & -5.61 & -5.95 \\
Sc II & -9.41 & -9.36 \\
Ti II & -7.10 & -7.16 \\
V II & -8.04 & -8.08 \\
Cr I & -6.26 & -6.37 \\
Cr II & -6.34 & -6.39 \\
Mn I & -6.84 & $\ldots$ \\
Mn II & -6.54 & -6.62 \\
Fe I & -4.81 & -4.75 \\
Fe II & -4.71 & -4.69 \\
Ni II & -5.91 & -6.10 \\
Sr II & -9.52 & -9.52 \\
Ba II & -10.07 & -9.71 \\
\hline$\tau$ Her & & \\
Species & Paper III & This paper \\
\hline He I & -1.04 & -0.96 \\
C II & -3.63 & -3.53 \\
N II & -4.30 & -4.09 \\
O II & $-2.76:$ & -3.36 \\
Mg II & -4.65 & -4.60 \\
Al II & $\ldots$ & -5.82 \\
Al III & -5.79 & -5.59 \\
Si II & -4.57 & -4.49 \\
Si III & -4.52 & -4.42 \\
S II & -5.01 & -4.76 \\
Ca II & -5.93 & -5.74 \\
Ti II & -7.39 & -6.76 \\
Cr II & $-6.74:$ & -6.55 \\
Fe II & -4.80 & -4.72 \\
Fe III & -4.63 & -4.62 \\
Ni II & -7.15 & -6.72 \\
\hline & & \\
\hline & & \\
\hline
\end{tabular}

analyses confirm the consistency and the quality of the entire series.

This study's abundances are compared with those of the Sun (Grevesse et al. 1996) in Table 7. For 16 elements the mean underabundance of $\alpha$ Dra with respect to the Sun is $-0.23 \pm 0.26$ dex. Thus the typical metallic abundance for this star is about $60 \%$ of solar.

$\tau$ Her's mean underabundance for 12 elements (excluding $\mathrm{Ni}$ ) is $-0.07 \pm 0.13$ which is $85 \%$ of solar. However, $\mathrm{Ni}$ is underabundant by a factor of 10 , but this is based only on one line. The agreement in abundances from two stages of ionization for $\mathrm{Al}, \mathrm{Si}$, and $\mathrm{Fe}$ is acceptable when one considers the number of lines involved. That He appears is have a slightly greater than solar abundance might reflect
Table 7. Comparison of derived and solar abundances $(\log N / \mathrm{H})$

\begin{tabular}{|c|c|c|c|c|c|}
\hline Species & $\alpha$ Dra & $\tau$ Her & $\gamma$ Lyr & HR 7926 & Sun \\
\hline He I & $-1.06 \pm 0.08$ & $-0.96 \pm 0.02$ & $-0.98 \pm 0.05$ & $-1.00 \pm 0.02$ & -1.01 \\
\hline C I & -3.78 & $\ldots$ & $\ldots$ & $\ldots$ & -3.45 \\
\hline C II & $-3.67 \pm 0.28$ & $-3.53 \pm 0.28$ & $-3.61 \pm 0.27$ & $-3.48 \pm 0.25$ & -3.45 \\
\hline N II & $\ldots$ & $-4.09 \pm 0.17$ & $\ldots$ & -4.24 & -4.03 \\
\hline O I & -3.50 & $\ldots$ & $-3.20 \pm 0.25$ & $-3.29 \pm 0.17$ & -3.13 \\
\hline O II & $\ldots$ & -3.36 & $\ldots$ & $\ldots$ & -3.13 \\
\hline Mg I & $-4.54 \pm 0.17$ & $\ldots$ & $-4.57 \pm 0.19$ & $\ldots$ & -4.42 \\
\hline Mg II & $-4.70 \pm 0.30$ & $-4.60 \pm 0.03$ & $-4.60 \pm 0.19$ & $-4.54 \pm 0.07$ & -4.42 \\
\hline $\mathrm{Al} \mathrm{I}$ & $-6.24 \pm 0.22$ & $\ldots$ & $-6.43 \pm 0.02$ & $\ldots$ & -5.53 \\
\hline Al II & -5.83 & -5.82 & $\ldots$ & -5.78 & -5.53 \\
\hline Al III & $\ldots$ & $-5.59 \pm 0.09$ & $\ldots$ & $-5.28 \pm 0.27$ & -5.53 \\
\hline Si II & $-4.96 \pm 0.19$ & $-4.49 \pm 0.04$ & $-4.45 \pm 0.27$ & $-5.00 \pm 0.27$ & -4.45 \\
\hline Si III & $\cdots$ & $-4.42 \pm 0.18$ & $\ldots$ & $-4.39 \pm 0.07$ & -4.45 \\
\hline S II & -4.35 & $-4.76 \pm 0.18$ & $\ldots$ & $-4.89 \pm 0.23$ & -4.67 \\
\hline $\mathrm{Ca} \mathrm{I}$ & -6.25 & $\ldots$ & $\ldots$ & $\ldots$ & -5.64 \\
\hline Ca II & -5.95 & -5.74 & -6.40 & -5.45 & -5.64 \\
\hline Sc II & $-9.36 \pm 0.11$ & $\ldots$ & -9.97 & $\ldots$ & -8.83 \\
\hline Ti II & $-7.16 \pm 0.20$ & $-6.76 \pm 0.18$ & $-7.54 \pm 0.21$ & $-7.15 \pm 0.17$ & -6.98 \\
\hline V II & $-8.08 \pm 0.23$ & $\ldots$ & -8.63 & $\ldots$ & -8.00 \\
\hline $\mathrm{Cr} \mathrm{I}$ & $-6.37 \pm 0.12$ & & $\ldots$ & $\ldots$ & -6.33 \\
\hline Cr II & $-6.39 \pm 0.16$ & $-6.55 \pm 0.19$ & $-6.48 \pm 0.19$ & $-6.58 \pm 0.26$ & -6.33 \\
\hline Mn II & -6.62 & $\ldots$ & -6.44 & $-6.07 \pm 0.16$ & -6.61 \\
\hline Fe I & $-4.75 \pm 0.25$ & $\ldots$ & $-4.90 \pm 0.25$ & $\ldots$ & -4.50 \\
\hline Fe II & $-4.69 \pm 0.18$ & $-4.72 \pm 0.16$ & $-4.84 \pm 0.19$ & $-4.66 \pm 0.22$ & -4.50 \\
\hline Fe III & $\ldots$ & $-4.62 \pm 0.07$ & $\ldots$ & $-4.49 \pm 0.20$ & -4.50 \\
\hline Ni II & $-6.10 \pm 0.22$ & -6.72 & $-6.01 \pm 0.19$ & $-6.03 \pm 0.28$ & -5.75 \\
\hline Sr II & $-9.52 \pm 0.01$ & $\ldots$ & $-9.86 \pm 0.25$ & $\ldots$ & -9.03 \\
\hline Ba II & $-9.71 \pm 0.10$ & $\ldots$ & $\ldots$ & $\ldots$ & -9.87 \\
\hline
\end{tabular}

undetected blending lines in those lines with $\mathrm{He} / \mathrm{H}$ values $\geq 0.11$.

For $\gamma$ Lyr, the mean underabundance for 15 elements is $-0.39 \pm 0.40$ which is $40 \%$ of solar. $\mathrm{Al}, \mathrm{Ca}, \mathrm{Sc}$, and Sr are underabundant by more than 0.85 dex while Ti and $\mathrm{V}$ are about 0.6 dex underabundant. Although the underabundances of $\mathrm{Ca}$ and $\mathrm{Sc}$ are typical of the metalliclined $(\mathrm{Am})$ stars, the general abundance pattern is not (Adelman et al. 1997). But $\gamma$ Lyr is more luminous and evolved than the classical Am stars. It also shows more rotation than most stars which were studied in this temperature range. Perhaps it is a star seen equator-on which evolved from such a star.

The mean underabundance for HR 7926 from 13 elements is $-0.15 \pm 0.10 \mathrm{dex}$. This does not include the value for $\mathrm{Mn}$ which is based on two weak lines and is overabundant. The pattern of other abundance anomalies suggest we should consider this identification to be tentative.

Acknowledgements. SJA thanks Dr. James E. Hesser, Director of the Dominion Astrophysical Observatory for the observing time. His contribution to this paper was supported in part by grants from The Citadel Development Foundation. This research was supported in part by the Research Fund of the University of Istanbul, project number OR-61/280699. 


\section{References}

Abt, H. A., \& Morrell, N. I. 1995, ApJS, 99, 135

Adelman, S. J. 1987, PASP, 99, 515

Adelman, S. J. 1988, MNRAS, 230, 671 (Paper III)

Adelman, S. J. 1994, MNRAS, 271, 355 (Paper XIII)

Adelman, S. J. Gulliver, A. F., \& Rayle, K. E. 2001, A\&A, 367, 597 (Paper XXIV)

Adelman, S. J., Caliskan, H., Kocer, D., \& Bolcal, C. 1997, MNRAS, 288, 470

Biemont, E., Grevesse, N., Faires, L. M., et al. 1989, A\&A, 209, 391

Blackwell, D. E., Shallis, M. J., \& Simmons, G. J. 1982, MNRAS, 199, 33

Breger, M. 1976, ApJS, 32, 7

Campos, A. J. 1980, ApJ, 238, 250

Cowley, A. 1972, AJ, 77, 750

Dworetsky, M. M. 1971, Ph.D. Thesis, University of California at Los Angeles

ESA 1997, The Hipparcos and Tycho Catalogs, SP-1200

Fuhr, J. R., Martin, G. A., \& Wiese, W. L. 1988, J. Phys. Chem. Ref. Data, 17, Suppl. 4

Fuhr, J. R., \& Wiese, W. L. 1990, in CRC Handbook of Chemistry and Physics, ed. D. R. Lide (CRC Press, Cleveland, $\mathrm{OH}$ )

Grevesse, N., Noels, A., \& Sauval, A. J. 1996, in Cosmic Abundances, ed. S. Holt, \& G. Sonneborn (San Francisco, Astron. Soc. Pacific), ASP Conf. Ser., 99, 117

Guthrie, B. N. G. 1985, MNRAS, 216, 1

Gulliver, A. F., Hill, G., \& Adelman, S. J. 1996, in 5th Vienna Symposium on Stellar Atmospheres and Spectrum Synthesis, ed. S. J. Adelman, F. Kupka, \& W. W. Weiss (San Francisco, Astron. Soc. Pacific), ASP Conf. Ser., 108, 232

Hauck, B., \& Mermilliod, M. 1980, A\&AS, 40, 1

Hill, G., Fisher, W. A., \& Poeckert, R. 1982, Publ. Dom. Astrophys. Obs. Victoria, 16, 27

Hubeny, I., Lanz, T., \& Jeffrey, C. S. 1994, Daresbury Lab. New. Anal. Astron. Spectra, No. 20, 30

Huldt, S., Johansson, S., Litzen, U., \& Wyart, J.-F. 1982, Phys. Scripta, 25, 401
Iglesias, L., \& Velasco, R. 1964, Publ. Inst. Opt. Madrid, No. 23

Johansson, S. 1978, Phys. Scripta, 18, 217

Jönsson, G., Kröll, S., Persson, A., \& Svanberg, S. 1984, Phys. Rev. A, 30, 2429

Kharitonov, A. V., Tereshchenko, V. M., Knyazeva, L. N., Shityuk, I. S., \& Glushkova, E. A. 1997, Baltic Astron., 6,585

Kurucz, R. L. 1979, ApJS, 401

Kurucz, R. L. 1993, Atlas 9 Stelllar Atmosphere Programs and $2 \mathrm{~km} / \mathrm{s}$ grid, Kurucz CD-Rom No. 13, Smithsonian Astrophysical Observatory (Cambridge, MA)

Kurucz, R. L., \& Bell, B. 1995, Atomic Data for Opacity Calculations, Kurucz CD-Rom No. 23, Smithsonian Astrophysical Observatory (Cambridge, MA)

Kurucz, R. L., \& Avrett, E. H. 1981, SAO Special Report, No. 391

Lanz, T., \& Artru, M.-C. 1985, Phys. Scripta, 32, 115

Lawler, J. E., \& Dakin, J. T. 1989, JOSA B, 6, 1457

Lemke, M. 1989, A\&A, 225, 125

Martin, G. A., Fuhr, J. R., \& Wiese, W. L. 1988, J. Phys. Chem. Ref. Data, 17, Suppl. 3

Mitchell, S. A. 1909, ApJ, 30, 239

Moore, C. E. 1945, A Multiplet Table of Astrophysical Interest (Princeton University Observatory)

Napiwotzki, R., Schönberner, D., \& Wenske, V. 1993, A\&A, 268,653

Reader, J., \& Corliss, C. H. 1980, NSRDS-NBS 68, Part 1, US Government Printing Office (Washington, DC)

Schild, R., Peterson, D. M., \& Oke, J. B. 1971, ApJ, 166, 95

Wiese, W. F., Fuhr, J. R., \& Deters, T. M. 1996, J. Phys. Chem. Ref. Data, Monograph 6

Wiese, W. L., \& Martin, G. A. 1980, NSRDS-NBS 68, Part 2, US Government Printing Office (Washington, DC)

Wiese, W. L., Smith, M. W., \& Glennon, B. M. 1966, NSRDSNBS 4, US Government Printing Office (Washington, DC)

Wiese, W. L., Smith, M. W., \& Miles, B. M. 1969, NSRDS-NBS 22, US Government Printing Office (Washington, DC)

Wolff, S. C. 1978, ApJ, 222, 556 